(C) Elsevier Sequoia S.A., Lausanne - Printed in The Netherlands

\title{
MODULE FOR RAPID NORMAL AND INVERSE NORMAL PULSE. POLAROGRAPHY: APPLICATION TO STUDY OF ADSORPTION
}

\author{
TIMOTHY E. CUMMINGS \\ University of Miami, Coral Gables, FL 33124 (U.S.A.)
}

WILLIAM T. BRESNAHAN, SUK YOUN SUH and PHILIP J. ELVING

Department of Chemistry, University of Michigan, Ann Arbor, MII 48109 (U.S.A.)

(Received 27th February 1979; in revised form 11th June 1979)

\begin{abstract}
Construction and utilization of a module for interfacing with a rapid response potentiostat are described, which permits use of normal pulse polarography (NPP) and inverse normal pulse polarography (INPP). In trace analysis by NPP, the module permits micromolar detection. INPP is shown to be very useful for adsorption studies, e.g., the relative surface excess $(\Gamma)$-potential profile can be rapidly obtained. The influence of time between pulse application and current sampling (discharge time, $t_{d}$ ) on signal level and response profile is discussed. A $t_{d}$ as short as $0.57 \mathrm{~ms}$ was tested for both techniques; shorter $t_{\mathrm{d}}$ intervals may be possible. The electronic operation is described in sufficient detail to allow ready alteration of the circuitry shown for interfacing to most potentiostats. Applicability of the module is illustrated by examination of the adsorption of adenine at the mercury electrode/ aqueous solution interface.
\end{abstract}

\section{INTRODUCTION}

Recently, the application of an in-house built potentiostat [1] to the investigation of the adsorption of adenine using cyclic voltammetry, ac polarography and inverse normal pulse polarography (INPP), was described [2]; the rapid response of the potentiostat, which considerably exceeds the response capabilities of commercially available instruments, is important to studies of weak adsorption [2], as demonstrated by the time-dependence of response in Flemming's work [3]. INPP involves drop birth and growth at a potential, which varies very slowly with time, and, then, application of a sudden pulse to a potential which remains constant throughout the entire potential range of the experiment; the latter potential is one at which the faradaic reaction of interest occurs. The current flow, after decay of or correction for the charging current produced on the pulse application, is a measure of the amount of electroactive species adsorbed at the potential preceding the pulse application. Because of the evident capabilities of INPP for adsorption studies, particularly when used in conjunction with a rapid-response potentiostat, and the lack of instrumentation suitable for automatic application of the requisite waveform [2], a module has been designed, which can be easily interfaced to a potentiostat and which 
permits automatic application of the INPP waveform.

Normal pulse polarography (NPP) involves drop birth and growth at a constant potential with pulses to potentials which vary throughout the scan. Since the INPP waveform is basically the inverse of that used in NPP, capability to perform NPP has been incorporated into the module. Use of a rapid response potentiostat for NPP permits an appreciable increase in signal over that attainable using commercial instrumentation, thus offering even lower detection limits for trace analysis.

\section{STRUCTURE AND OPERATION}

Essential requirements of the module are: (a) ability to apply the required rapid rise-time and quick settling voltage pulse to the potentiostat; (b) capability of varying the discharge time, $t_{\mathrm{d}}$, i.e., time between pulse application and current measurement; (c) capability properly to sequence temporally the applied pulse with respect to the sample-and-hold and drop-knocker functions of the potentiostat; (d) ability to apply any voltage pattern shown in Fig. 1 as may be necessary for study of either oxidation or reduction processes using INPP and/or NPP. The first characteristic is important because all pulse polarographic methods are essentially potential-step techniques. The second requirement is important because the signal-to-noise ratio of pulse methods is gener-

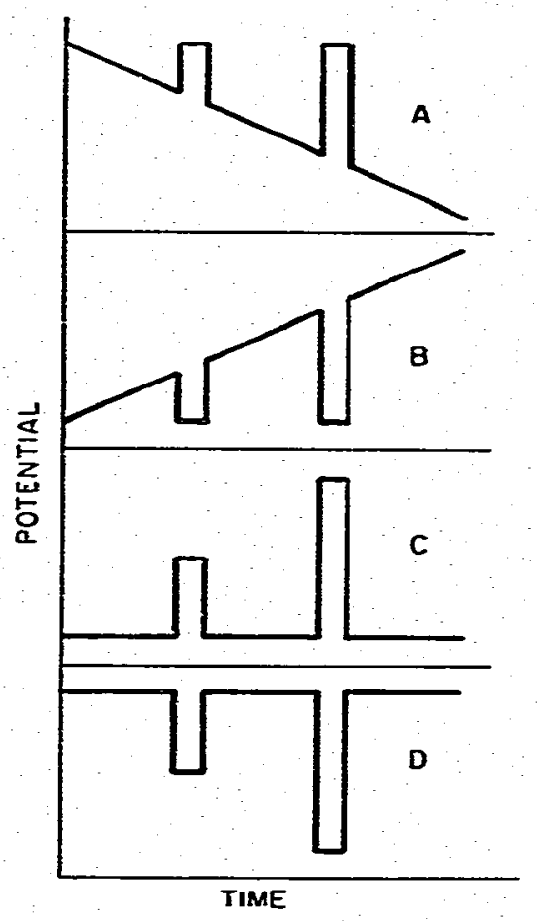

Fig- 1. Voitage patterns available from the module. A, inverse normal pulse polarography (INPP) for oxidation. B, INPP for reduction. $C$, normal pulse polarography (NPP) for oxidation. $D$, NPP for reduction. Time scale is distorted. 
ally improved by using the shortest feasible discharge time; the latter is generally the time at which the charging current due to the pulse becomes negligible.

\section{Module organization}

The pulse module consists of three major functional units: (a) timing circuitry for proper sequencing of pulse application with timed functions of the potentiostat, i.e., current sampling and drop dislodgment; (b) analog integrator, which supplies the proper potential scan rate to the recorder and to the solidstate switch for transmission to the potentiostat; (c) the solid-state switch and associated operational amplifier unity gain inverter, the former of which transmits the voltage pulse to the potentiostat only at the proper times and the latter of which is used for negative voltage ramps, since only positive voltage polarities are transmittable by the described switch. A simplified schematic diagram of the module is given in Fig. 2; a detailed circuit diagram and parts list can be obtained from the authors.

\section{Timing circuitry}

The timing circuit in the potentiostat (1) consists of three monostable multivibrators (MS I-III) with low stable states. The MS logic sequences are shown in Fig. 3 .

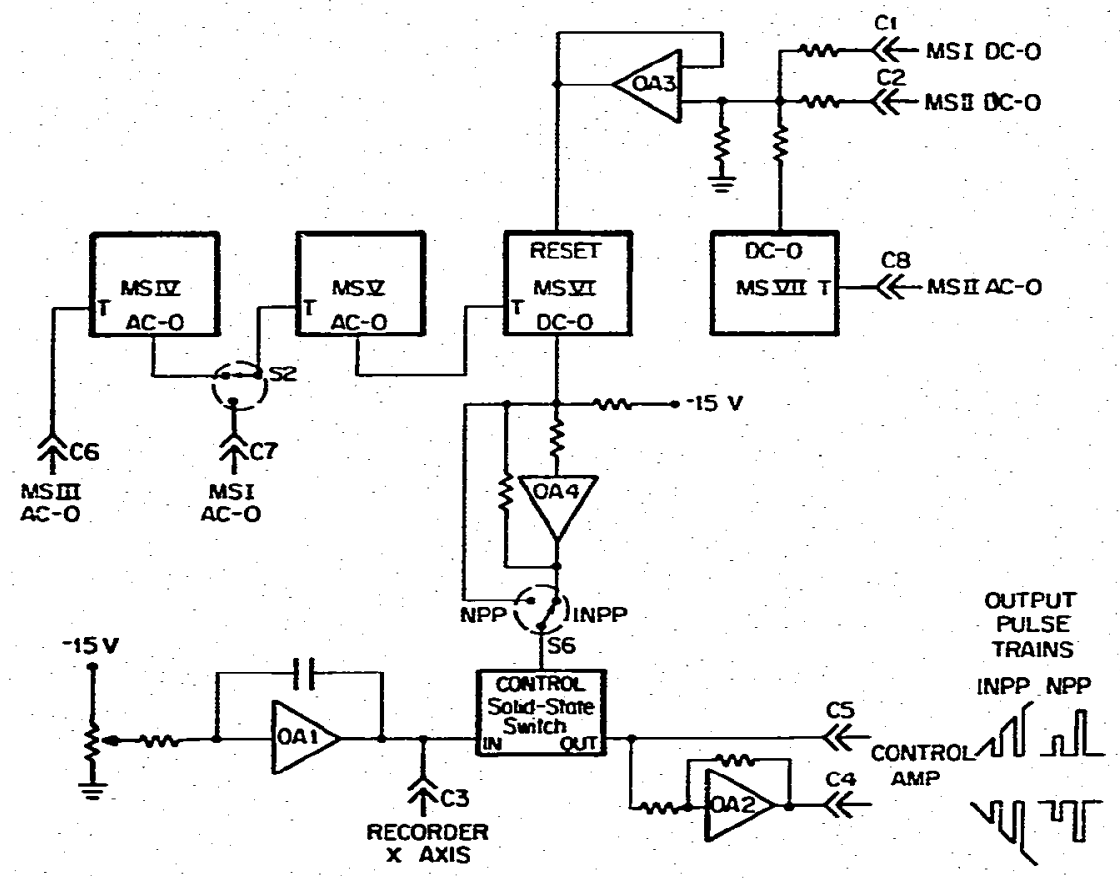

Fig. 2. Simplified schematic diagram of the pulse polarographic module. $O A=$ operational amplifier, $\mathrm{MS}=$ monostable multivibrator, $\mathrm{SX}=\operatorname{switch} \mathrm{X}, \mathrm{CX}=\mathrm{BNC}$ connector $\mathrm{X}, \mathrm{DC}-\mathrm{O}=$ de-coupled output, $A C-O=$ ac-coupled output, $\mathbf{T}=$ trigger input. 
MS: LEVELS SET IN THE POTENTIOSTAT

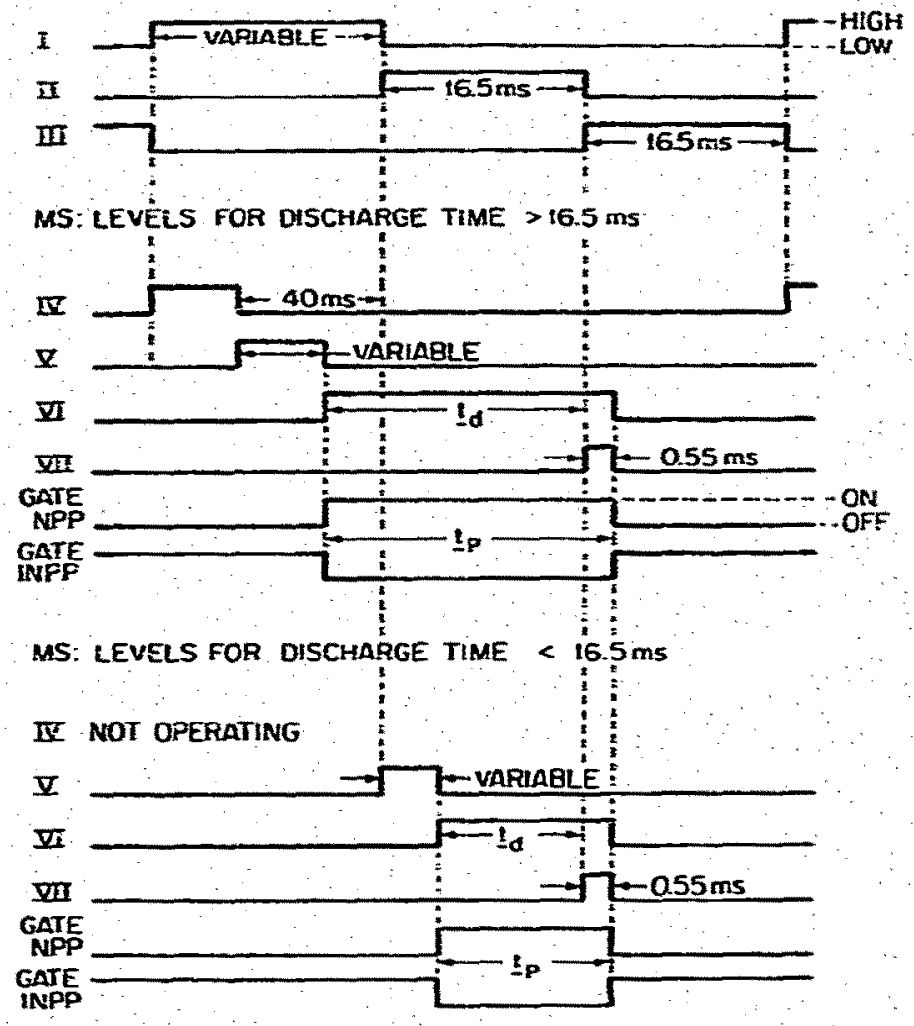

Fig. 3. Logic sequences for the monostable (MS) mu!tivibrators I through VII as functions of the discharge time $\left(t_{d}\right)$, i.e., time between pulse application and current sampling. The high and low levels for the first MS and the on and off conditions for the first gate (solid-state switch) are shown; these levels and conditions are similar for the other.MS and gates.

The high-time of MS I determines the length of the drop life $(1,2,3$ or $5 \mathrm{~s})$; its failing output triggers MS II which controls the condition of the sample and hold amplifier $(\mathrm{S} / \mathrm{H})$. When MS II is high, the $\mathrm{S} / \mathrm{H}$ samples the output of the current-followe: amplifier of the potentiostat; when MS II goes low, the signal at that instant is held. MS II also triggers MS III which fires the drop-dislodger and retriggers MS I, thus; starting a new cycle. The high-times of MS II and III. are set at $16.5 \mathrm{~ms}$.

The pulse moduie was constructed to accomodate the potentiostat's timing circuit [1]; the design is not unique and could easily be modified to suit the needs of the user. The module operates by generating a pulse at a praselected time, i.e., $t_{d}$, before the $\mathrm{S} / \mathrm{H}$ acquires a signal. The present design uses two timing sequences, one each for $t_{\mathrm{d}}$ values greater than and less than the 16.5-ms high time of MS II. Each pulse cycle consists of a trigger from the potentiostat's timer which initiates a delay preset in the module, after which the pulse is applied to the potentiostat. Since the delay is initiated by the same sequence 
which controls the $\mathrm{S} / \mathrm{H}$, pulse application and current measurement are synchronized.

For $t_{\mathrm{d}}>16.5 \mathrm{~ms}$ (S2 is counterclockwise (ccw) in Fig. 2), MS IV is triggered simultaneously with MS I. MS IV, which goes low 40 ms before MS II goes high, triggers MS V which is the preset delay prior to pulse application. The high-time of MS V is variable and deternines $t_{d} ; t_{\mathrm{d}}=56.5 \mathrm{~ms}-$ (MS V hightime). When MS V goes low, MS VI is triggered and the pulse is applied via control of a solid-state switch with MS VI.

For $t_{\mathrm{d}}<16.5 \mathrm{~ms}$, (S2 is clockwise (cw) in Fig. 2), MS IV is not used and MS $V$ is triggered by MS I. MS $V$ and MS VI have the same functions as when $t_{\alpha}>$ $16.5 \mathrm{~ms}$, but here $t_{\mathrm{d}}=16.5 \mathrm{~ms}-$ (MS $\mathrm{V}$ high-time). For any $t_{\mathrm{d}}$ value, the pulse is terminated using the reset function of MS VI. The reset is accomplished by summing fractions of MS I, II and VII outputs; MS VII, whose high-time is set at $0.55 \mathrm{~ms}$, is triggered by the falling edge of MS II. When MS $\mathrm{i}, \mathrm{II}$ and VII are low, the cutput of OA-3 is low and MS VI is reset. The pulse is terminated in this manner so that the duration of the pulse is $0.55 \mathrm{~ms}$ larger than $t_{\mathrm{d}}$, allowing the $\mathrm{S} / \mathrm{H}$ to acquire a signal before the voltage pulse to the electrode is terminated.

A range of $t_{\mathrm{d}}$ varying from 0.57 to $36.6 \mathrm{~ms}$ was examined; shorter discharge times may be usable in some cases.

\section{Sample and hold amplifier}

The accuracy and dynamic response characteristics of the $\mathrm{S} / \mathrm{H}$ are crucial in rapid response pulse polarography. The $\mathrm{S} / \mathrm{H}$ in the potentiostat (Hybrid Systems $725 \mathrm{LH}$ ) has a gain of $1 \pm 0.01 \%$, an acquisition time $( \pm 10 \mathrm{~V})$ of $10 \mu \mathrm{s}$, a slew rate of $1.5 \mu \mathrm{s}^{-1}$ and a aperture time of $100 \mathrm{~ns}$. These characteristics are more than sufficient to (a) follow the current-time transients obtained in the pulse polarographic experiments, and (b) acciuire a signal with the desired accuracy in time and magnitude at submillisecond $t_{d}$ values.

\section{Selection of NPP or INPP}

The essential difference between NPP and INPP is that, for NPP, the potential during most of the drop life, i.e., the prepolarization patential $E_{\mathrm{pp}}$, is held constant during the entire experiment, while the pulse magnitude, which defines the potential to which the potentio:stat is pulsed, $E_{\mathrm{p}}$, changes with each drop; for INPP, $E_{\mathrm{pp}}$ changes with each drof, while $E_{\mathrm{p}}$ is constant during the experiment. The constant potential, i.e., $E_{p_{p},}$ for NPP or $E_{p}$ for INPP, is set using the initial-potential circuit of the potentiostat.

For NPP, $\mathbf{S 6}$ is set to its ccw position; the $n$, the solid-state switch is ON when MS VI is high. For the majority of the drop-life, the MS VI output is low and the switch is OFF; hence, the cell poten tial equals the potentiostat's initial potential circuit value, $E_{\mathrm{pp}}$. During the last few milliseconds of the drop-life, the switch is ON and the output voltage of CAA 1 will either be summed to (C5 for oxidation studies) or subtracted from (C4 for reduction studies) the potentiostat's initial potential to yield the pulse potential, $E_{p}$.

For INPP, S6 is set to its cw position; then the output of MS VI is summed 
with $-15 \mathrm{~V}$, the sum is inverted by $O A 4$, and the result is applied to the switch control. For most of the drop-life, MS VI is low and the switch is ON. During this period, the OA 1 output is summed (C5) or subtracted (C4) from the potentiostat's initial potential, $E_{\mathrm{p}}$, to yield the prepolarization potential, $E_{\mathrm{pp}}$. When MS VI goes high during the last few milliseconds of the drop-life the voltage levels at $\mathrm{C4}$ and $\mathrm{C5}$ are essentially zero. Thus, during this period, only $E_{\mathrm{p}}$ is applied to the cell.

\section{Selection of pulse scan rate and polarity}

The rate at which $E_{\mathrm{pp}}$ changes for INPP and at which $E_{\mathrm{p}}$ changes from drop to drop for NPP is determined by the scan rate of the integrator, OA 1 . This scan rate depends upon the $O A 1$ input voltage, which is controlled by a potentiometer. $E_{\mathrm{p}}$ of NPP and $E_{\mathrm{pp}}$ of INPP are monitored by connecting the output of $O A 1$ (CB) to the $x$-axis of the recorder.

For NPP, the scan rate determines the rate of change of $E_{\mathrm{p}}$ from drop to drop and, it should be noted, it also determines a slight change in $E_{\mathrm{p}}$ during pulse application. For example, for a $5-\mathrm{mV} \mathrm{s}^{-1}$ scan rate and a 2-s drop time, $E_{\mathrm{p}}$ will change $10 \mathrm{mV}$ drop to drop; for a pulse time of $5 \mathrm{~ms}, E_{\mathrm{p}}$ will change $25 \mu \mathrm{V}$ during pulse application. Although $25 \mu \mathrm{V}$ is negligibly small, with a high scan rate and long pulse time the change of $E_{\mathrm{p}}$ during pulse application may be significant. For INPP, the scan rate effect on $E_{\mathrm{pp}}$ is significant; since $E_{\mathrm{pp}}$ is applied during most of the drop-life, a $5-\mathrm{mV} \mathrm{s}^{-1}$ scan rate will alter $E_{\mathrm{pp}}$ by ca. $10 \mathrm{mV}$ during a 2-s drop-life. The effect is further discussed in the Evaluation of Module section (p. 81).

\section{EXPERIMENTAL}

\section{Chemicals}

Adenine was obtaired from National Biochemicals. Mchlvaine buffer, $\mathrm{pH} 4.85$ and $0.5 M$ ionic strength, was prepared from reagent grade chemicals [4]. Mercury for electrodes was chemically purified and distilled.

Solutions were decxygenated with purified $N_{2}$ for ca. 20 min; an $N_{2}$ atmosphere was maintained in the cell during experiments.

\section{Instrumentation}

The jacketted, one-chamber cell with Luggin capillary (cf. fig. 4 of ref. 1) was maintained at $25.00 \pm 0.05^{\circ} \mathrm{C}$. The Luggin capillary was positioned within one drop-diameter of the working electrode. The mean DME Hg-flow-rate was $1.15 \mathrm{mg} \mathrm{s}^{-1}$ at open circuit in the McIlvaine buffer.

The potentiostat has been described [1]. A Heath digital multimeter was used to monitor applied de potentials. Data were displayed on a Houston Model $2000 x-y$ recorder.

Reported potentials are vs. the saturated calomel electrode. 


\section{OPERATION AND PERFORMANCE}

The critical factors for performance of the module have been indicated (cf. Structure and Operation): The necessity for being able to vary the available $t_{\mathrm{d}}$ magnitude is due to both variation of the charging time with conditions and the desire to investigate variation of signal with time, e.g., to determine by extrapolation the faradaic signal on INPP at time zero due to reduction of an adsorbed species, so that the surface excess $(\Gamma)$ can be estimated. The shortest feasible $t_{\mathrm{d}}$ can be determined by observing on an oscilloscope the charging current-time relation at a $E_{\mathrm{p}}$ where no faradaic activity occurs; $t_{\mathrm{d}}$ is chosen where the charging current is negligible with respect to the faradaic current anticipated in the $E_{\mathrm{p}}$ region of interest.

\section{Normal pulse polarography}

NPP and corresponding dc polarographic behavior of adenine are shown in Fig. 4. The aic polarographic wave is not resolved from background discharge which is shifted to more pcsitive potential due to the catalytic influence of adenine on $\mathrm{H}^{+}$reduction [5].

(1) Effect of $E_{\mathrm{pp}}$ on wave-height. In the case of electroactive species which show. no tendency toward adsorption at the solution/mercury interface, the NPP wave-height will be independent of $E_{\mathrm{pp}}$, provided that $E_{\mathrm{pp}}$ is not in the region of faradaic activity of the species. For a species such as adenine, which shows potential-dependent adsorption $[2,3,6,7]$, the wave-height will depend upon $E_{\mathrm{pp}}$; however, this dependence will decrease with increasing $t_{\mathrm{d}}$ (cf. next sec-

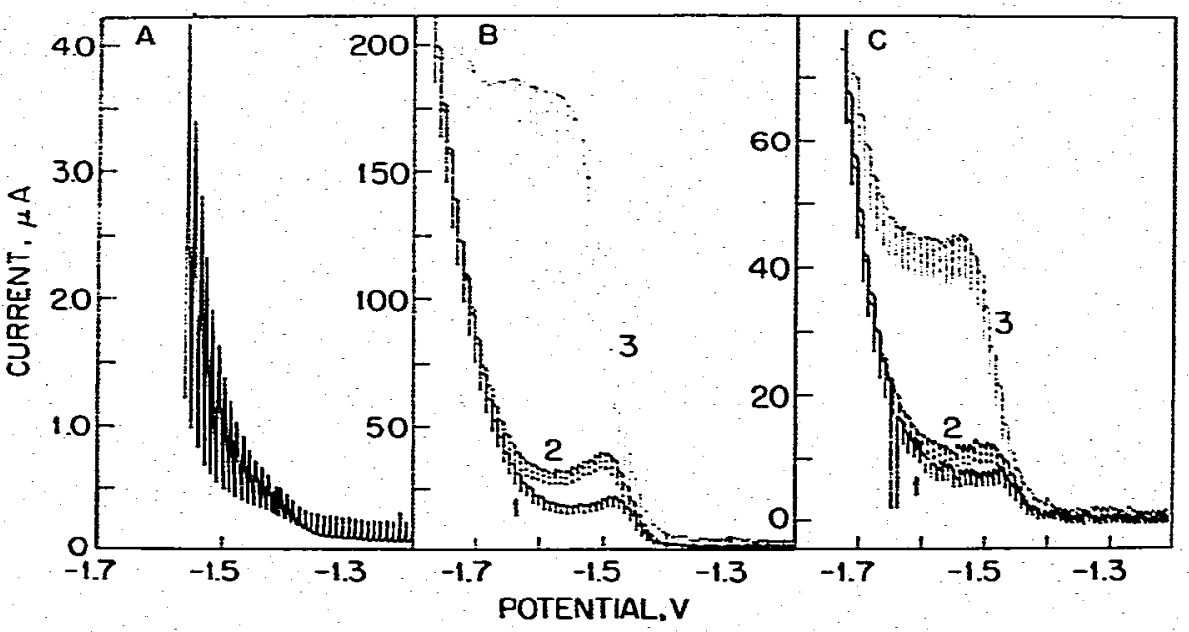

Fig- 4. Polarograms for 17.4 $\mu \mathrm{M}$ adenine at $\mathrm{pH}$ 4.85. (A) De polarogram at controlled 2-s drop-time. (B) Normal pulse polarogram at $E_{\mathrm{pp}}=-0.4 \mathrm{~V}$. Magnitude of $t_{\mathrm{d}}$ in ms: (1) 2.6 ; (2) 1.6 ; (3) 0.57 . (C) Normal pulse polarogram at $E_{\mathrm{pp}}=-0.9 \mathrm{~V}$. Magnitude of $t_{\mathrm{d}}$ in ms: (1) $2.6 ;$ (2) $1.6 ;(3) 0.57$. 
tion). Figs. $4 \mathrm{~B}$ and $C$ show the effect of $E_{\mathrm{pp}}$ on the adenine wave-height as a result of the smaller tendency toward adsorption of adenine at $-0.9 \mathrm{~V}$ as compared to $-0.4 \mathrm{~V}$ (cf. Fig. 5 and section on INPP).

(2) Effect of $t_{\mathrm{d}}$ on wave-height. The effect of $t_{\mathrm{d}}$ on wave height is marked. The height at $-1.5 \mathrm{~V}$ for $t_{\mathrm{d}}$ of $0.57 \mathrm{~ms}$ and $E_{\mathrm{pp}}$ of $-0.4 \mathrm{~V}$ is nearly six times that at $1.6 \mathrm{~ms}$ and eight times that at $2.6 \mathrm{~ms}$. The gain in sensitivity achievable with a short $t_{\mathrm{d}}$, when the electroactive species is adsorbed, is clear. Comparison of the wave-heights in Figs. $4 \mathrm{~B}$ and $\mathrm{C}$ yields qualitative information regarding the reduction rate of the adsorbed species. The considerably smaller extent of adsorption at $-0.9 \mathrm{~V}$ than at $-0.4 \mathrm{~V}$ is clearly shown by the much larger NPP response at $E_{\mathrm{pp}}$ of $-0.4 \mathrm{~V}$ than at $E_{\mathrm{pp}}$ of $-0.9 \mathrm{~V}$ for $t_{\mathrm{d}}$ of $0.57 \mathrm{~ms}$. Yet, at $t_{\mathrm{d}}$ of $1.6 \mathrm{~ms}$, the response for $E_{\mathrm{pp}}$ of $-0.4 \mathrm{~V}$ only slightly exceeds that for $-0.9 \mathrm{~V}$; for $t_{\mathrm{d}}$ of $2.5 \mathrm{~ms}$, the response difference for the two $E_{\mathrm{pp}}$ is negligible. Thus, by 1.6 $\mathrm{ms}$, virtually all of the adsorbed species has been reduced and the observed current is principally due to reduction of diffusing species.

For species not adsorbed at the electrode surface, the NPP wave height is definable from the Cottrell equation, since, under these circumstances, the NPP behavior is derived from the potential-step experiment. Thus, a $t_{d}$ of $0.57 \mathrm{~ms}$ should provide a five-fold inerease in signal over that obtainable with instruments having a fixed $t_{\mathrm{d}}$ of $16.5 \mathrm{~ms}$.

(3) Maximum effect in the presence of adsorption. For $E_{\mathrm{pp}}=-0.4 \mathrm{~V}$, a current "maximum" is present at $t_{\mathrm{d}}$ of 1.6 and $2.6 \mathrm{~ms}$, which is absent (or greatly diminished) for the same $t_{\mathrm{d}}$ at $E_{\mathrm{pp}}=-0.9 \mathrm{~V}$; the "maximum" is not seen at $t_{\mathrm{d}}=0.57 \mathrm{~ms}$ (Figs. $4 \mathrm{~B}$ and $\mathrm{C}$ ). The "maxima" are not related to the instrumentation and are not due to the convective stirring responsible for maxima of the first and second kind; they are a direct result of the reduction of an adsorbed reactant.

Anomalies in the shapes of normal pulse polarograms were first reported by Barker and Bolzan [8] who correctly proposed that the rate of decay of faradaic current upon pulse application was a function of the potential to which the electrode was pulsed when a reactant is adsorbed. At potentials in the vicinity of $E_{1 / 2}$, the reduction of an adsorbed layer is slow relative to its reduction at more negative potentials; thus, the current measured when $E_{\mathrm{p}}$ is near $E_{\mathrm{l} / 2}$ exceeds that measured when $E_{\mathrm{p}}$ is in the plateau region; hence, a current peak is recorded near $E_{\mathrm{g} / 2}$ -

The "Barker-Bolzan peaks" have been studied in detail by Flanagan et al. [9] who used digital simulation to generate normal pulse polarographic currentpotential curves when reactant and/or product adsorption is governed by either linear or non-linear isotherms. They stressed that depression in normal pulse polarographic limiting current occurs as a result of reactant adsorption. This depression of limiting current has serious analytical consequence because the linear relationship between limiting current and bulk concentration is lost.

Remarkably large "Barker-Bolzan peaks" are exhibited in the NPP records for the one-electron reduction of $\mathrm{NAD}^{+}$in which the adenine moiety is known to be responsibie for adsorption. A more detailed study of these peaks is in progress [10]. 
(4) Effect of $t_{\mathrm{d}}$ on background discharge. Since many background discharges are irreversible, the background discharge current amplitude relative to desired signal increases with $t_{\mathrm{d}}$. A special case of background discharge response dependence on $t_{\mathrm{d}}$ is the catalytic effect of pyrimidine and purine reduction products on $\mathrm{H}^{+}$reduction. The advantage of short drop-times for differential pulse polarographic determination of adenine, cytosine and their nucleosides has been established [11]; it is due to desreased catalytic $\mathrm{H}^{+}$reduction resulting from the smaller amounts of reduction products generated during short droptimes (0.5-1 s). In the case of NPP, for which electroactivity occurs only during $t_{d}$, the extent of catalytic $\mathrm{H}^{+}$reduction increases with increasing $t_{\mathrm{d}}$; hence, the background discharge relative to blank shifts positively with increasing $t_{\mathrm{d}}$ (cf. Fig. 4).

(5) Influence of drop-time. Drop-time only affects the ratio of signal-to-instrumentally generated noise; hence, increasing the drop-time should increase this ratio. The improved $S / N$ ratio is achieved at the expense of scanning time, since the number of points on the wave and on the plateau is related to the rate at which $E_{\mathrm{p}}$ changes in $\mathrm{mV} \mathrm{s}{ }^{-1}$; thus, for improved resolution, $E_{\mathrm{p}}$ should change less per drop. A compromise between drop-time and scan duration must be made, based on the sample being examined, e.g., need for resolution, expected or desired signal level, and maximum acceptable duration of scan.

\section{Inverted normal pulse polarography}

The INPP response (Fig. 5) shows a current $-E_{\mathrm{pp}}$ profile similar to those previously reported for low adenine concentrations [2,3]. At the present time, little or no theory is available for quantitative correlation between response and the surface excess, $\Gamma$; however, INPP is a rapid, powerful tool for studies of the relative dependence of $\Gamma$ on applied potential, since the response is proportional to $\Gamma$ at $E_{\mathrm{pp}}$.

As is evident from Fig. 4 , the choice of $E_{\mathrm{p}}$ is critical when resolution from another process is small. Under such circumstances, a dc polarogram should not be used to select $E_{p}$, since time-dependent factors may influence resolution, e.g., $-1.6 \mathrm{~V}$ is a potential for which response varies from being on the adenine plateau (curves 3 in Figs. $4 B$ and $C$ ) to being on the foot of background discharge (curves 2 of Figs. $4 \mathrm{~B}$ and $\mathrm{C}$ ) to being far into background discharge (Fig. $4 A$ ); hence, $E_{p}$ is best selected by examination of an NPP record at the intended $t_{\mathbf{d}}$.

(1) Anomalous behavior near $E_{1 / 2}$. There is a rapid drop in response for $E_{\mathrm{pp}}$ negative of $-1.35 \mathrm{~V}$ and a slow rise in response negative of $-1.46 \mathrm{~V}$ (Fig. 5 ). Reduction of adenine begins at ca. $-1.35 \mathrm{~V}$ (Fig. $4 \mathrm{~B}$ ); thus, for $E_{\mathrm{pp}}$ positive of $-1.35 \mathrm{~V}$, the surface concentration is determined by adsorption effects and the bulk concentration. For $E_{\mathrm{pp}}$ negative of $-1.35 \mathrm{~V}$, electrolysis of adenine occurs during the time of prepolarization, $t_{\mathrm{pp}}$, which is only negligibly different from the drop-time, $t$, i.e., $t-t_{\mathrm{d}}=t_{\mathrm{pp}}$ and $t_{\mathrm{d}}$ is at most several $\mathrm{ms}$. The resulting adenine consumption results in concentration polarization and lowers the surface concentration available for reduction when $E$ is stepped to $E_{\mathrm{p}}$; hence, the cur- 


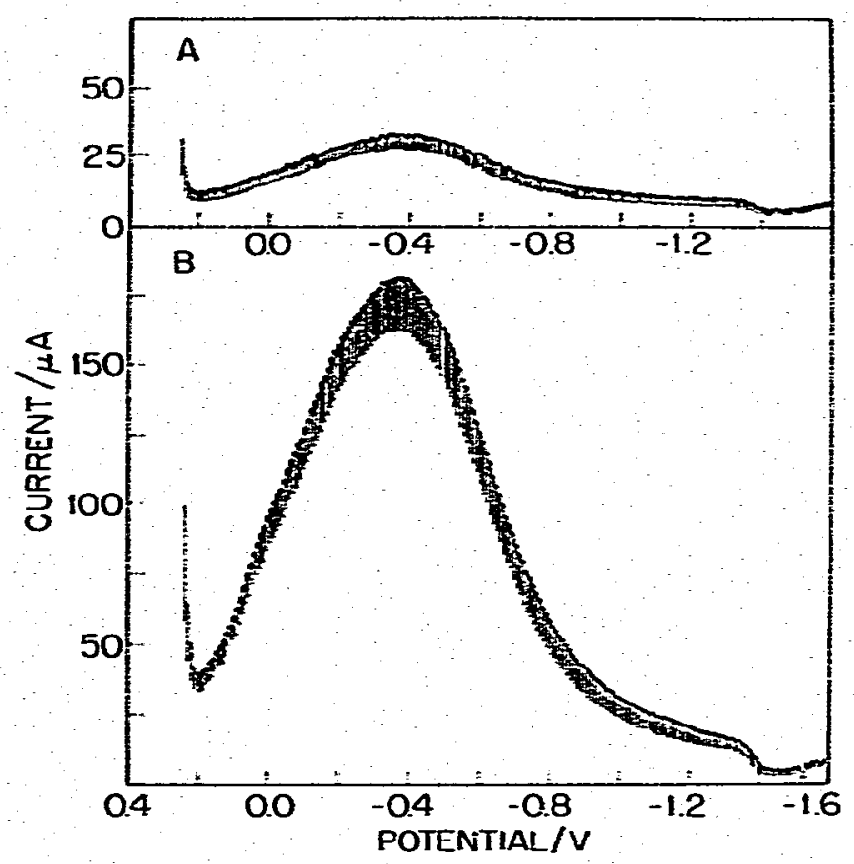

Fig. 5 . Inverted normal pulse polarograms for $17.4 \mathrm{mM}$ adenine at $\mathrm{pH} 4.85$. Potential axis represents $E_{\mathrm{pp}}$. Magnitude of $t_{\mathrm{d}}$ in ms: (A) 1.6 ; (B) 0.57 .

rent is lowered. As $E_{\mathrm{pg}}$ becomes increasingly negative, the amount of reduced species produced during $t_{\mathrm{pp}}$ increases as does the current at $E_{\mathrm{p}}$ due to catalytic $\mathbf{H}^{+}$reduction. In the case of adenine, the countering effects of decreasing adenine pulse current and increasing catalytic $\mathrm{H}^{+}$reduction current with more negative $E_{\mathrm{pp}}$ result in a change in net current trend at $-1.46 \mathrm{~V}$.

(2) Effect of $t_{\mathrm{d}}$ on response. Because the INPP signal is due to reduction of adscrbed material, whose rate is uncontrolled by diffusion, response decreases rapidly with increasing $t_{\mathrm{d}}$ (Fig. 5). In general, optimum response is achieved at the shortest possible $t_{\mathrm{d}}$, provided that $t_{\mathrm{d}}$ is longer than the period of double layer charging due to pulse application.

The apparent flatness of the $i-E_{\mathrm{pp}}$ peak at $-0.4 \mathrm{~V}$ for $t_{\mathrm{d}}$ of $1.6 \mathrm{~ms}$ as compared to that for $0.57 \mathrm{~ms}$ is deceptive. When the response at $-1.3 \mathrm{~V}$ is subtracted from the $i-E_{\mathrm{pp}}$ profile, ratios of response at $0.0,-0.2$ and $-0.6 \mathrm{~V}$ to peak response at ca. $-0.4 \mathrm{~V}$ are equal (within \pm 0.05 ) for both $t_{\mathrm{d}}$; thus, on the time scales employed, no chemical kinetic effects induce a $t_{\mathrm{d}}$-dependence on the $i-E_{\mathrm{pp}}$ profile for the current contribution from adsorbed species. This fact is important because only protonated adenine is reduced [5]; since unprotonated adenine is also present, it can be protonated to form the electroactive species. The kinetic effect would be visible on INPP only if the $\Gamma-E$ profiles for the conjugate acid and base differed and if the $t_{\mathrm{d}}$ range were sufficiently wide to observe protonation kinetics; however, based on studies of a measurement method for adsorbed state $\mathrm{p} K_{\mathrm{a}}[2,12]$, the adenine protonation reaction 
does not become significant for times shorter than ca. $2 \mathrm{~ms}$. Comparison of Fig. 5 with $i-E_{\mathrm{pp}}$ profiles for a $t_{\mathrm{d}}$ of $2.9 \mathrm{~ms}$ [2] shows that the profile at the longer $t_{\mathrm{d}}$ has a broad flat-topped region from ca. -0.2 to $-0.5 \mathrm{~V}$; this broader top probably reflects reduction of adenine adsorbed in the neutral form.

(3) Effect of drop-time. The drop-time dependence comments for NPP also apply to INPP, except that resolution is generally not a problem; however, the variation of $E_{\mathrm{pp}}$ during drop-life, i.e., scan rate, is critical in adsorption studies. Since the response is a measure of $\Gamma$ at $E_{\mathrm{pp}}$, the scan rate should be sufficiently slow that $E_{\mathrm{pp}}$ is relatively constant during a drop-life. On the other hand, INPP can be advantageously used to examine the kinetics of adsorption as manifest in a drop-time dependence of the current density $-E_{\mathrm{pp}}$ profile.

\section{EVALUATION OF MODULE}

In conjunction with a rapid response potentiostat, the pulse module is capable of yielding a response at least an order-of-magnitude greater than that achievable with most commercial instrumentation.

In the presence of adsorbable species which are electroactive within the potential range involved, it is apparent that the observed NPP and INPP responses will be grossly dependent on the experimental factors which affect adsorption, e.g., the presence and concentration in solution of surface-active species, composition of the supporting electrolyte and its ionic strength, concentration of the adsorbate of interest, and temperature; where slow adsorption-desorption processes are involved, time-scale of the experiment will be a factor. The $0.5 \mathrm{M}$ McIlvaine buffer of $\mathrm{pH} 4.85$ used in the present study to illustrate the use of the pulse module, has been frequently used in examining the electrochemical behavior of the nucleic acid bases and their nucleoside and nucleotide derivatives.

A distinct advantage of INPP is that it involves use of a step from a state which is better equilibrated than can be achieved with NPP. Although the use of a controlled drop-time, which is limited by the natural drop frequency in the case of a dme, limits the equilibration time available, there is no reason why a hanging mercury drop electrode or even a solid constant area electrode could not be used, since the final process in INPP "cleans" the electrode surface if the redox products are not surface-active.

Normal pulse polarography

An advantage of the present unit is the ability, using short $t_{\mathrm{d}}$, to improve resolution of the wave from background discharge when the reduction products catalyze the latter. Additionally, the shorter $t_{\mathrm{d}}$ can theoretically yield a signal enhancement of greater than ten. For ultra-trace assay, a slower sample-andhold amplifier with less output droop rate is recommended; the one eroployed in the present study can respond to high frequency noise, which can increase the noise level. Nonetheless, with the present instrumentation and $t_{\mathrm{d}}$ of $0.57, \mathrm{a}$ detection limit $(S / N=2)$ for adenine of $0.3 \mu M$ or less is attainable for $E_{p p}=$ $-0.9 \mathrm{~V}$ and $0.1 \mu M$ or less for $E_{\mathrm{pp}}=-0.4 \mathrm{~V}$; the latter corresponds to $7 \times 10^{-8} \mathrm{~g}$ adenine for a $5-\mathrm{ml}$ volume. 
The output droop rate of the present sample-and-hold $\left(15 \mathrm{mV} \mathrm{s}^{-1}\right)$ becomes noticeable at recorder sensitivities exceeding $100 \mathrm{mV}^{-1}$. Less droop would improve the appearance and the ease of reading the polarograms.

Examination of the current in the absence of faradaic activity is helpful in defining the minimum acceptable $t_{\mathrm{d}}$. On decreasing $t_{\mathrm{d}}$, a time will be observed at which the current begins to increase rapidly. This time represents that at which the double layer is essentially charged vis $a$ vis the potential step; $t_{d}$ should be longer than that required for this double layer charging step.

\section{Inverted normal pulse polarography}

The response capabilities for INPP are excellent. The technique is capable of rapidly yielding the relative $\Gamma-E_{\text {pp }}$ profile, which, in contrast to ac polarographic and capacitance bridge measurements, can be useful in discoverirg the presence of both faradaically active and inactive adsorbed species. Additionally, qualitative adsorption kinetics studies can be performed easily and rapidly by variation of the drop-time and $t_{\mathrm{d}}$.

\section{Alternative circuitry}

Although the module described was designed for use with a previously reported potentiostat [1], the electronic design is sufficiently simple to permit modification of the circuit for compatibility with other potentiostats. The basic requirements for the potentiostat are the presence of timing circuitry for a sample-and-hold amplifier and drop-knocker, and a source for input of the pulse signal.

(1) Timing circuit connections for long $t_{\mathrm{d}}$. With controlled drop-time and sample-and-hold available on a potentiostat, at least three monostable multivibrators, operating in sequence, are required; their functions are identical to those described for MS I-III [1] and will be referenced by these labels. The MS I to III outputs must be made available to the pulse module. In general, each MS output's stable state will be ca. $0 \mathrm{~V}$; its metastable state will be +5 to +15 V. If the MS have metastable states which are at negative voltage, which is incompatible with the MS used in the present module, then modifications are necessary.

Two types of interfacing are required: (a) direct connection or dc-coupled; (b) connection through a small capacitor, i.e., ac-coupled. For the potentiostatmodule combination described, the ac coupling requirements are available from the potentiostat; if this is not true for another potentiostat, ac coupling can be easily built into the module.

(2) Pulse signal input. Some commercial potentiostats have an external input for auxiliary signals; if one is available, then either $\mathbf{C 4}$ or $\mathbf{C 5}$, depending upon desired pulse polarity, is connected to it. Which connection must be made for a given polarity may vary from one potentiostat to another, depending upon the potentiostat's circuitry; hence, choice of the proper connection must be experimentally determined. 
Since the rise-time of the pulse applied to the cell will depend upon the potentiostat, the characteristics of $O A 2$ can be matched to the potentiostat's. Although OA 2 should have a rise-time at least as fast as the potentiostat, it would be wasteful to install an operational amplifier as $O A 2$ which is.faster than the potentiostat.

\section{ACKNOWLEDGMENTS}

The authors thank the National Science Foundation, which helped support the work described. WTB thanks the Horace H. Rackham School of Graduate Studies of the University of Michigan for a fellowship.

\section{REFERENCES}

1 T.E. Cummines. M.A. Jensen and P.J. Elving. Electrochim. Acta. 23 (1978) 1173.

2 A1.A. Jensen. T.E. Cummings and P.J. Elving. Bioelectrochem. Bioenarg-, 4 (1977) 447.

3 J. Flemming. J. Electroanal. Chem., 75 (1977) 421.

4 P.J. Elving. J.T. Markouitz and I. Rosenthal. Anal. Chem.. 28 (1956) 1179.

5 D.L. Smith and P.J. Elving. J. Amer. Chem. Soc., 84 (1962) 1412.

6 M. Katz. T.E. Cummings and P.J. Elving. Ber. Bunsenges. Phys. Chem.. 83 (1979) 614.

7 H. Konoshita, S.D. Christian, M.H. Kim, J.G. Barker and G. Dryhurst in D.T. Sawyer (Ed.), Electrochemical Studies of Biological Systems, American Chemical Society, Washington. 1977.

8 G.C. Barker and J.A. Bolzan. Z. Anal. Chem.. 216 (1966) 215.

9 J.B. Flanagan. K. Takahashi and F.C. Anson. J. Electroanal. Chem.. 85 (1977) 257.

10 .W.T. Bresnahan and P.J. Elving, work in progress.

11 T.E. Cummings, J.R. Fraser and P.J. Elving, ms, in prep.

12 T.E. Cummings and P.J. Elving, ms. in prep. 